\title{
RESTORATION OF ENMAP DATA THROUGH SPARSE RECONSTRUCTION
}

\author{
Daniele Cerra, Jakub Bieniarz, Tobias Storch, Rupert Müller, and Peter Reinartz \\ German Aerospace Center (DLR) \\ Remote Sensing Technology Institute (IMF) \\ 82234 Wessling, Germany
}

\begin{abstract}
This paper presents the first results of applying sparse reconstruction methods to restore a simulated dataset for the Environmental Mapping and Analysis Program (EnMAP), the forthcoming German spaceborne hyperspectral mission. Each image element is independently decomposed using contributions from a limited number of pixels, which come directly from the image and have previously undergone a low-pass filtering in noisy bands. Thus, the denoising application is reduced to a weighted sparse unmixing problem. A first assessment of the results is encouraging as the original bands taken into account are reconstructed with a high Signal-toNoise Ratio and low overall distortions.
\end{abstract}

Index Terms - EnMAP, denoising, spectral unmixing, sparse reconstruction.

\section{INTRODUCTION}

The future EnMAP (Environmental Mapping and Analysis Program; www.enmap.org) mission will be able to acquire images at $\pm 30^{\circ}$ off-nadir to achieve revisit times of up to 4 days. The different acquisition angles and illumination conditions will introduce considerable variations in Signalto-Noise Ratio (SNR) across the spectral bands, which could benefit from denoising techniques with a high degree of automation. This paper proposes a modified version of Unmixing-based Denoising (UBD) [1], a denoising technique based on spectral unmixing [2], to selectively retrieve corrupted bands which may be useful for a given application. A novel algorithm derives from coupling UBD with sparse reconstruction algorithms, in order to increase its automation level and improve the denoising results in terms of a higher similarity to a model noise-free image. Weighting parameters are set in order to derive most of the information for the reconstruction of a given spectral band from other correlated bands. First results are presented on a noisy synthetic EnMAP dataset in which the proposed algorithm is able to successfully restore the corrupted band of interest. Comparisons with some well known algorithms suggest that the proposed technique could offer a viable solution for EnMAP images acquired in unfavourable conditions.
The paper is structured as follows. Section 2 gives a brief reminder on the EnMAP mission. Section 3 adopts sparse reconstruction methods to increase the degree of automatization and improve results from UBD, and Section 4 reports some experiments on a simulated EnMAP hyperspectral dataset. We conclude in Section 5.

\section{THE ENMAP MISSION}

EnMAP is a German, earth observing, imaging spectroscopy, spaceborne mission planned for launch in 2018 and with a lifetime of five years [3]. It addresses hyperspectral remote sensing with the major objectives of measuring, deriving, and analysing parameters on the status and evolution of terrestrial and aquatic ecosystems on a global scale. Applications comprise agriculture, forest, geology, urban, and coastal themes. The HSI (hyperspectral imager) will consist of two pushbroom imaging spectrometers: one for the VNIR (visible and near infrared) spectral range from 420 to $1000 \mathrm{~nm}$ with a sampling of $6.5 \mathrm{~nm}$, and one for the SWIR (shortwave infrared) spectral range from 900 to $2450 \mathrm{~nm}$ with a sampling of 10 $\mathrm{nm}$. The ground pixel size will remain constant at certain latitude, i.e. $30 \times 30 \mathrm{~m}$ at nadir at $48^{\circ}$ northern latitude. With 1000 valid pixels this yields to a swath width of $30 \mathrm{~km}$. One of the key system performance parameters is the SNR (in this paper considered as the power ratio between signal and background noise). Figure 1 illustrates the predicted performance for SNR at the sensors for nadir observations under three different conditions and for $10 \mathrm{~nm}$ equivalent bandwidth [4].

Thus, even if the SNR is predicted to be high typically, for situations of low surface albedo or sun zenith angle it will be reduced and methods for de-noising of hyperspectral images become essential.

\section{UNMIXING-BASED DENOISING AND SPARSE RECONSTRUCTION}

Unmixing-based Denoising (UBD) exploits spectral unmixing results to selectively recover bands affected by a low SNR in hypespectral images [1]. The output of the unmixing process, which aims at decomposing each image element in sig- 

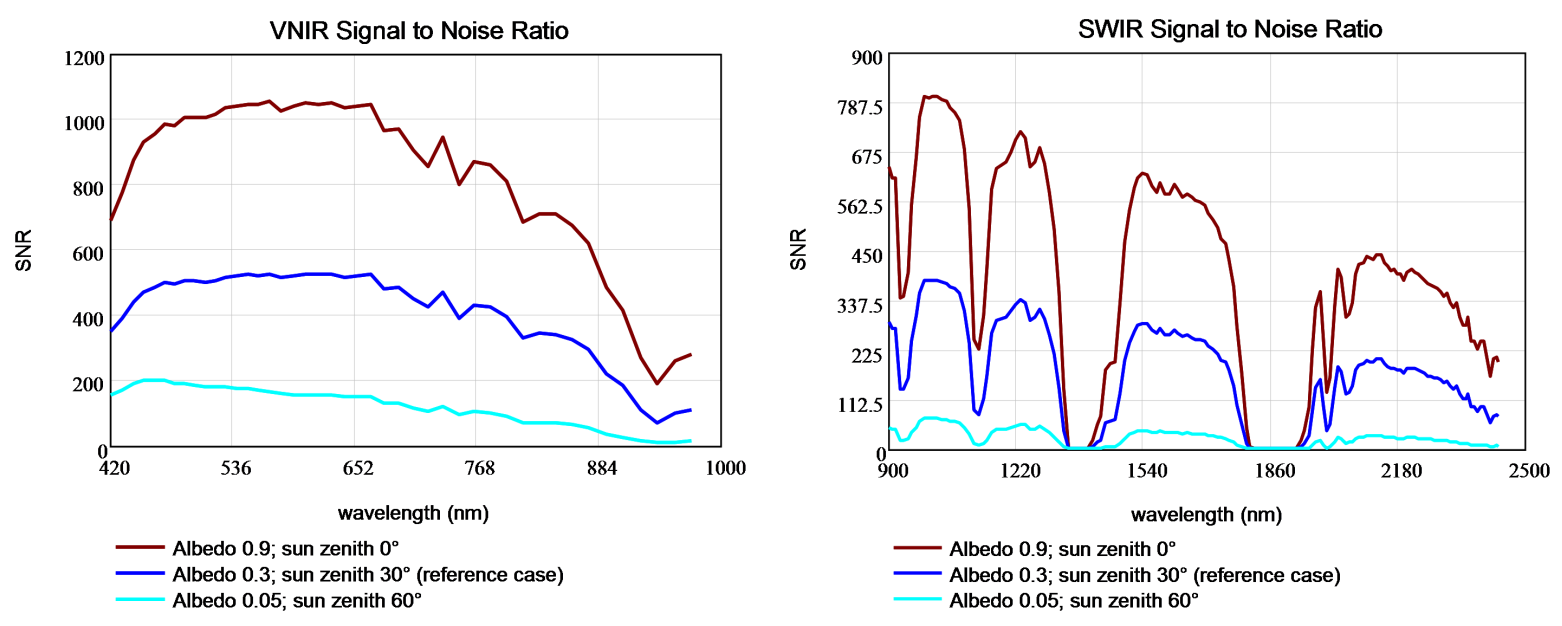

Fig. 1: Predicted performance for SNR (Signal to Noise Ratio), Courtesy of OHB System AG [4].

nals typically related to pure materials [2], is inferred into the reconstruction of a given noisy band, ignoring the residual vector which is mainly characterized by undesired noise. One of the problems of UBD is that the spectral library of interest must be known a priori. As in the general case this is not true, the library must be initialized by extracting with a method of choice a restricted number of reliable reference spectra as pure as possible. Afterwards, spectra are iteratively added by selecting areas in the error images related to the reconstruction of a band of interest, in a similar way to the Iterative Error Analysis (IEA) end-member extraction algorithm [2]. This step can be time-consuming and subjective with several parameters to set, such as the number of reference spectra to extract or the maximum distortion allowed in the reconstruction. To solve these problems, the use of sparse reconstruction methods is proposed to skip the reference spectra selection step.

UBD can be related to sparse methods, if we consider that in its applications sparseness is enforced by considering the reference spectra as a sparsifying basis for the original highdimensionality dataset. It is interesting that in [1] the advantages of using Non-negative Least Squares (NNLS) as unmixing algorithm, which promotes sparsity in the abundance vectors, are discussed.

A redundant, over-complete spectral library $A$ is composed by a very large number of randomly selected image elements, in which the noisy bands are spatially smoothed in order to have a reliable value in homogeneous regions. Afterwards, each image element $y$ and the library $A$ are fed to a non-negative Basis Pursuit reconstruction algorithm [5], which guarantees a sparse solution by solving the following minimization problem:

$$
\min _{x}|A x-y|_{2}^{2}+\lambda|x|_{1} \text { subject to } x \geq 0,
$$

where $\lambda$ is the regularization parameter controlling the sparsity of the solution vector $x$, which contains the fractional

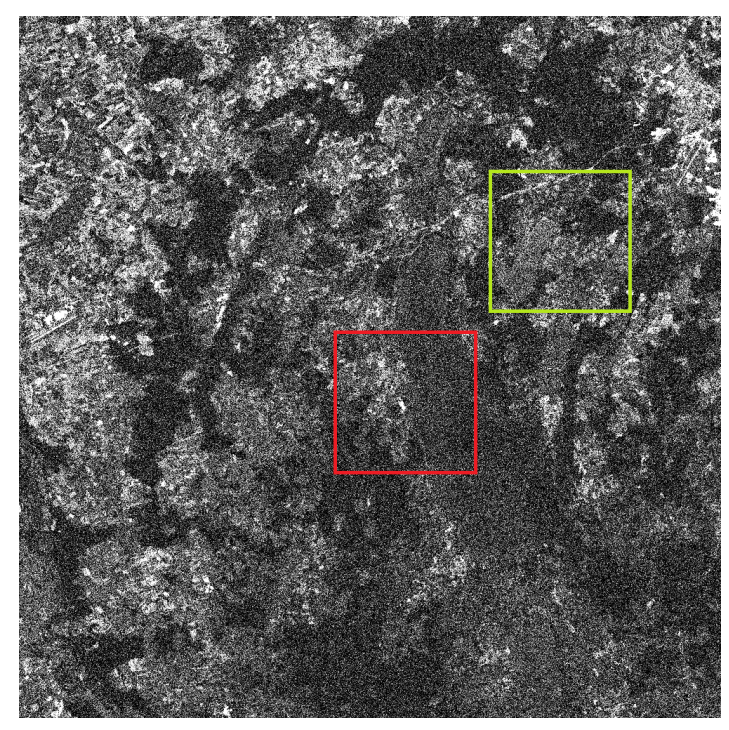

Fig. 2: Band 1 from the synthetic EnMAP Alpine Foreland dataset of size $1000 \times 1000$. In the green and red squares the details reported in Fig. 3.

abundances of the spectra selected in the reconstruction of $y$.

As this method aims at selectively retrieving corrupted spectral bands rather than trying to denoise the full hyperspectral dataset, a tuned weighting across the spectral bands is expected to yield better results. This ensures that the reconstruction process is mainly driven by spectral bands highly correlated with the band of interest. The problem becomes then:

$$
\min _{x}|w A x-w y|_{2}^{2}+\lambda|x|_{1} \text { subject to } x \geq 0,
$$

where $w$ is the weighting vector quantifying the relevance of each spectral band in the reconstruction process. 


\section{EXPERIMENTS}

We analyse the Alpine Foreland EnMAP dataset of size $1000 \times 1000$ pixels, which has been simulated with different SNR levels from applying water, vegetation and soil physical models to a Landsat image acquired on the area around lake Starnberg, Germany (for more information on the simulated dataset see [6]). We use the image with the worse average SNR equal to 100, which would be the worst case among the ones reported in Fig. 1, of which band 1 at $423 \mathrm{~nm}$ is depicted in Fig. 2. This case of study is not simple as all the bands have the same low SNR, unlike traditional HS datasets in which the SNR drastically increases whenever atmospheric absorption effects become less important. The denoising is carried out as described in eq. 2, with the spectral bands weighted according to their correlation with the band of interest, and $10 \%$ of the image elements selected to initialize the over-complete spectral library.

Results on two image subsets localized by the squares in Fig. 2 are reported in Fig. 3. The denoised images are very similar to the noise-free simulated dataset. We report quantitative quality parameters and comparisons with alternative methods in Table 1 as follows. We compare the results of the described approach (with and without weighting of the spectral bands in the reconstruction step) with a 3D implementation of Non-local Means denoising [7] and Minimum Noise Fraction (MNF) with manual selection of the best number of components, a hard parameter to set [8]. The figures of merit are Normalized Root Mean Square Error, expressed in percentage (best value: 0\%), Structure Similarity (SSIM) [9] (best value: 1), and SNR (best value: $\infty$ ). Even though an adaptation of SSIM for HS images has not been agreed yet (see [10]), we are taking into account a single band, making this assessment of particular interest. The known distortions of the noisy band are reported for reference. The method is also fast in terms of running time, taking 70.47 seconds on a standard laptop machine with 8 GB RAM and Intel(R) Core(TM) i5-2520M $2.50 \mathrm{GHz}$ processor to denoise the one million pixels with 224 spectral bands.

\begin{tabular}{|l|c|c|c|}
\hline Method & NRMSE (\%) & SSIM & SNR \\
\hline Noisy band at 420 nm & 8.64 & 0.207 & 100 \\
UBD - WSR & $\mathbf{2 . 0 3}$ & $\mathbf{0 . 6 7 8}$ & $\mathbf{1 8 9 2}$ \\
UBD - SR & 2.37 & 0.636 & 1361 \\
MNF (best result) & 2.46 & 0.526 & 926 \\
Wiener (best result) & 3.76 & 0.334 & 570 \\
3D Non-Local Means & 3.62 & 0.316 & 587 \\
\hline
\end{tabular}

Table 1: Comparison of average NRMSE, SSIM and SNR values for the denoising of the band reported in Fig. 2. UBD - WSR and UBD - SR stand for UBD with weighted sparse reconstruction and sparse reconstruction, respectively.

\section{CONCLUSIONS}

This paper tested a new denoising technique based on sparse reconstruction on simulated EnMAP data. The algorithm improves on the idea of Unmixing-based Denoising (UBD) by increasing its automation degree and by tuning the contribution of each spectral band to the final result. First results and comparisons with other techniques are satisfactory and could help in correcting EnMAP images acquired under unfavourable illumination conditions or at higher off-nadir looking angles. Furthermore, the operational fully-automatic onground processing which delivers standardized products to the international user community is expected to introduce at most $1 \%$ dead or bad pixels [11]. As UBD has been successfully tested on destriping and bad pixels restoration problems [12], the proposed method could be employed also to decrease the impact of such missing or corrupted data. In the experimental section the best weighting parameters have been manually selected, but they could be easily computed as a function of the spectral correlation with the band which is selected to retrieve and the SNR of each band. In order to achieve that, it is needed to perform a noise estimation step beforehand.

\section{Acknowledgements}

Simulated EnMAP dataset produced by VISTA, Munich and processed by Karl Segel, GFZ, Potsdam.

\section{REFERENCES}

[1] D. Cerra, R. Müller, and P. Reinartz, "Noise reduction in hyperspectral images through spectral unmixing," Geoscience and Remote Sensing Letters, IEEE, vol. 11, no. 1, pp. 109-113, Jan 2014.

[2] J. M. Bioucas-Dias, A. Plaza, N. Dobigeon, M. Parente, Q. Du, P. Gader, and J. Chanussot, "Hyperspectral unmixing overview: Geometrical, statistical, and sparse regression-based approaches," IEEE Journal of Selected Topics in Applied Earth Observations and Remote Sensing, vol. 5, no. 2, pp. 354-379, 2012.

[3] T. Stuffler, K. Foerster, S. Hofer, M. Leipold, B. Sang, H. Kaufmann, B. Penne, A. Mueller, and Christian Chlebek, "Hyperspectral Imaging - an Advanced Instrument Concept for the EnMAP Mission (Environmental Mapping and Analysis Program)," Acta Astronautica, vol. 65, no. 7, pp. 1107-1112, 2009.

[4] B. Sang, J. Schubert, S. Kaiser, V. Mogulsky, C. Neumann, K. P. Förster, S. Hofer, T. Stuffler, H. Kaufmann, A. Müller, T. Eversberg, and C. Chlebek, "The EnMAP hyperspectral imaging spectrometer: instrument concept, calibration, and technologies," in Proc. SPIE, 2008, vol. 7086. 

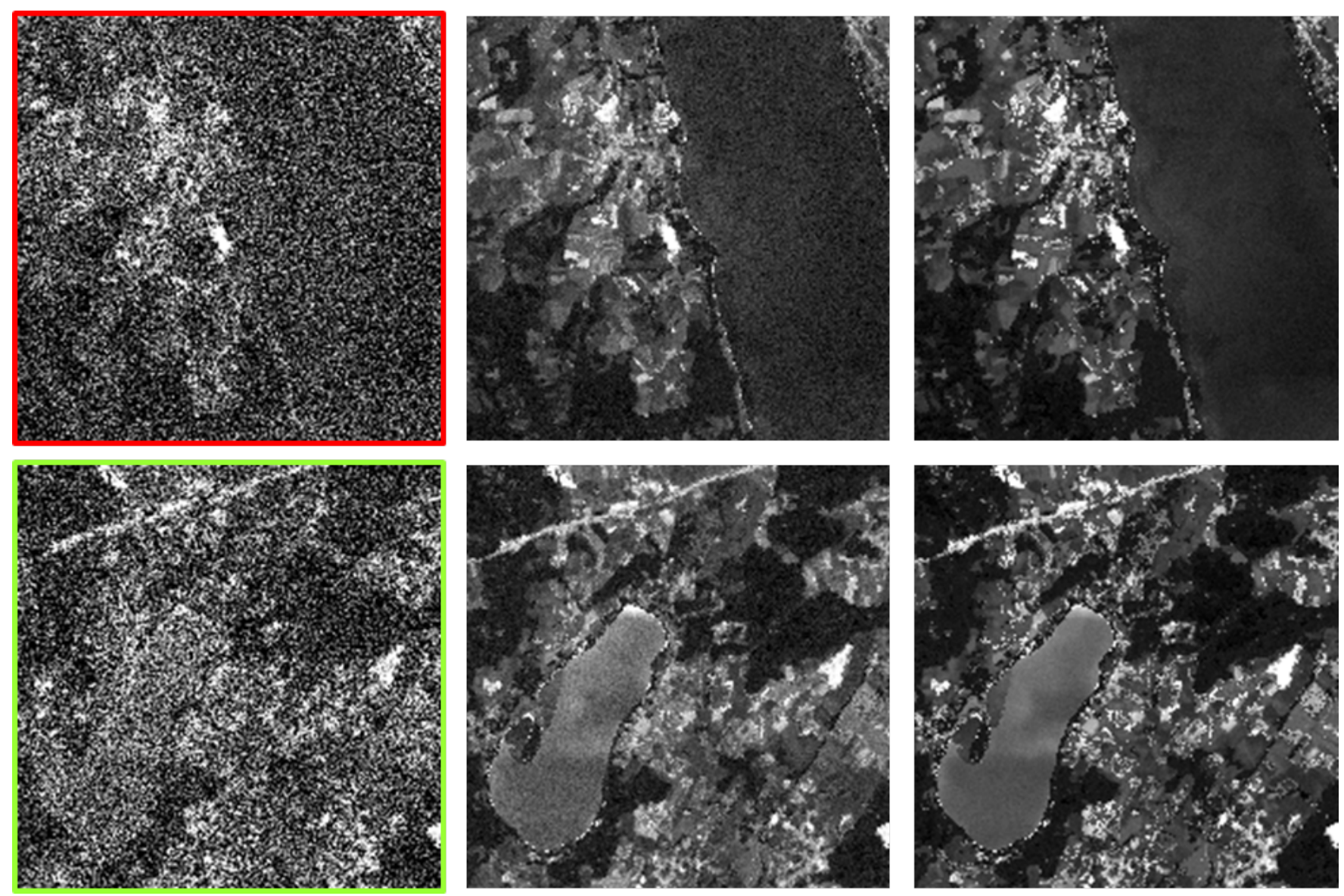

Fig. 3: Left: Zoomed details represented by the red and green squares in fig. 2. Center: Denoising results. Right: Subsets of the ideal noise-free image.

[5] S. S. Chen, D. L. Donoho, and M. A. Saunders, "Atomic decomposition by basis pursuit," SIAM Rev., vol. 43, no. 1, pp. 129-159, Jan. 2001.

[6] W. Verhoef and H. Bach, "Coupled soil-leaf-canopy and atmosphere radiative transfer modeling to simulate hyperspectral multi-angular surface reflectance and TOA radiance data," Remote Sensing of Environment, vol. 109, pp. 166-182, 2007.

[7] A. Buades, B. Coll, and J.M. Morel, "A non-local algorithm for image denoising," in CVPR'05, 2005, vol. 2 of CVPR '05, pp. 60-65.

[8] U. Amato, R. Cavalli, A. Palombo, S. Pignatti, and F. Santini, "Experimental approach to the selection of the components in the minimum noise fraction," IEEE Transactions on Geoscience and Remote Sensing, vol. 47, no. 1-1, pp. 153-160, 2009.

[9] Z. Wang, A. C. Bovik, H. R. Sheikh, and E. P. Simoncelli, "Image quality assessment: From error visibility to structural similarity," IEEE Transactions on Image Processing, vol. 13, no. 4, pp. 600-612, 2004.

[10] Y. Zhao, J. Yang, Q. Zhang, L. Song, Y. Cheng, and Q. Pan, "Hyperspectral imagery super-resolution by sparse representation and spectral regularization," EURASIP Journal on Advances in Signal Processing, vol. 2011, no. 1, pp. 87, 2011.

[11] T. Storch, M. Bachmann, S Eberle, M. Habermeyer, C. Makasy, A. de Miguel, H. Mühle, and R. Müller, "EnMAP Ground Segment Design: An Overview and Its Hyperspectral Image Processing Chain," Lecture Notes in Geoinformation and Cartography, pp. 49-62, 2013.

[12] D. Cerra, R. Müller, and P. Reinartz, "Unmixing-based denoising for destriping and inpainting of hyperspectral images," in IGARSS 2014, July 2014, pp. 4620-4623. 